\title{
Effects of a Chinese formula Salviae Miltiorrhizae and Ligustrazine hydrochloride solution injection in the acute ischemic stroke patients
}

\author{
Yuta Kobayashi ${ }^{1}$, Gangli Yan ${ }^{2}$, Rong Zhang ${ }^{2}$, Wenjuan Zuo ${ }^{2}$, Ruwei Wang ${ }^{2,3}$ \\ ${ }^{1}$ Faculty of Medicine, Shimane University, Japan, ${ }^{2}$ Guizhou Baite Pharmaceutical Corporation, China, ${ }^{3}$ Zhejiang \\ CONBA Pharmaceutical \& Drug Research Development Corporation, China
}

\section{Background}

Clinical treatment against acute ischemic stroke remains limited. Highly effective approaches to stroke treatment, such as systemic reperfusion and mechanical thrombectomy as well as tissue-plasminogen activator cannot be performed in the majority of patients. Neuroprotective strategies, i.e. prevention of irreversible neuron damage due to the ischemia, may improve stroke outcomes. However, only few pharmacological agents demonstrated clinical efficacy.

Methods

129 acute ischemic stroke patients were randomly divided into trial group $(\mathrm{n}=65)$ and the standard control group $(\mathrm{n}=$ 64). Ages were 40 to 80 years. As their neurological deficit, National Institute of Health stroke scale (NIHSS) score was evaluated between 3 points to 15 points. The treatment protocol was approved by the medical ethics committee and informed consent was gotten. According to the same test program trials were done in three centers at the same time, with the double-blind test. The safety analysis was evaluated in all cases. As the trial DL injection (a Chinese formula Salviae Miltiorrhizae and Ligustrazine hydrochloride solution; Guizhou Beit Pharmaceutical Co. Ltd. Chinese medicine Zhunzi H52020959) was infused intravenously, $10 \mathrm{ml} /$ time / day for $7 \sim 10$ days. As the standard control group: citicoline sodium injection (Anhui Lian Yi Pharmaceutical Co., Ltd.) was applied to control patients with intravenous infusion, 0.25 $\mathrm{g} /$ day for $7 \sim 10$ days. Rankin score (disability score) and NIHSS were evaluated.

Results

NIHSS scores and Rankin scores improved significantly by both drugs. There was no significant difference between the two groups in NIHSS score and Rankin score $(\mathrm{P}>0.05)$.

Conclusion

Salviae miltiorrhizae and ligustrazine hydrochloride injection is effective, safe, convenient and well tolerated in the treatment of patients with acute ischemic stroke. 